\title{
Defatted kenaf seed meal (DKSM) : prospective edible flour from agricultural waste with high antioxidant activity
}

\begin{abstract}
The present study reports nutritional composition, phenolic content and antioxidant activity of defatted kenaf seed meal (DKSM) in comparison to wheat, rice and sweet potato flours. Proximate analysis revealed that DKSM was high in protein $(26.19 \mathrm{~g} / 100 \mathrm{~g}$ DKSM) and carbohydrate (57.09 g/100 g DKSM). Magnesium, potassium and phosphorus were the major minerals (>1 g/100 g DKSM) found in DKSM through Energy Dispersive X-ray Spectrometric analysis. DKSM also exhibited appreciably higher total phenolic (3399.37 $\mu \mathrm{g}$ gallic acid equivalent (GAE)/g defatted material) and flavonoid contents (251.00 $\mu \mathrm{g}$ rutin equivalent (RE)/g defatted material) as well as antioxidant activity as compared to all selected edible flours $(\mathrm{p}<0.05)$. Gallic acid, (+)-catechin, 4-hydroxybenzoic acid, vanillic acid and syringic acid were determined as the predominant phenolics in DKSM through HPLC-DAD analysis. On the basis of this study, DKSM may be declared and commercialized as a highly antioxidative and nutritive edible flour, which can be prospectively used in the development of natural food preservative, nutraceuticals and functional foods.
\end{abstract}

Keyword: Hibiscus cannabinus L.; Defatted kenaf seed meal; Alternative edible flour; Antioxidant activity; Phenolic compounds 